\title{
Stochastic Modeling of Nonlinear Circuits via SPICE-Compatible Spectral Equivalents
}

\author{
Paolo Manfredi, Student Member, IEEE, Dries Vande Ginste, Senior Member, IEEE, \\ Daniël De Zutter, Fellow, IEEE, Flavio G. Canavero, Fellow, IEEE
}

\begin{abstract}
This paper presents a systematic approach for the statistical simulation of nonlinear networks with uncertain circuit elements. The proposed technique is based on spectral expansions of the elements' constitutive equations ( $\mathrm{I}-\mathrm{V}$ characteristics) into polynomial chaos series and applies to arbitrary circuit components, both linear and nonlinear. By application of a stochastic Galerkin method, the stochastic problem is cast in terms of an augmented set of deterministic constitutive equations relating the voltage and current spectral coefficients. These new equations are given a circuit interpretation in terms of equivalent models that can be readily implemented in SPICE-type simulators, as such allowing to take full advantage of existing algorithms and available built-in models for complex devices, like diodes and MOSFETs. The pertinent statistical information of the entire nonlinear network is retrieved via a single simulation. This approach is both accurate and efficient with respect to traditional techniques, such as Monte Carlo sampling. Application examples, including the analysis of a diode rectifier, a CMOS logic gate and a low-noise amplifier, validate the methodology and conclude the paper.
\end{abstract}

Index Terms-Circuit design, circuit simulation, nonlinear circuits, polynomial chaos, SPICE, statistical analysis, uncertainty.

\section{INTRODUCTION}

The increasing miniaturization of electronic equipment is amplifying the impact of tolerances and uncertainties on circuit performance. The variability has several sources, ranging from the manufacturing process to temperature fluctuations and aging, and requires the electrical response to be addressed from a statistical standpoint. A common practice to handle this inherent randomness in circuit simulators is to use Monte Carlo [1] or similar sampling-based methods. A sufficiently large set of different scenarios is generated according to the statistical properties of the random parameters and simulated to collect samples of the output response. Despite its simplicity, the main drawback lies in the large number of instances that must be considered, thus often making the computational time prohibitive. To speed-up the design phase, engineers have been seeking for more efficient techniques (e.g. [2]).

Great attention has been recently attracted by the framework of the polynomial chaos (PC) theory [3]. Leveraging PC, random quantities and their governing equations are expanded

P. Manfredi and F. Canavero are with the EMC Group, Department of Electronics and Telecommunications, Politecnico di Torino, 10129 Torino, Italy (e-mail: paolo.manfredi@polito.it; flavio.canavero@polito.it).

D. Vande Ginste and D. De Zutter are with the Electromagnetics Group, Department of Information Technology, Ghent University, 9000 Gent, Belgium (e-mail: dries.vandeginste@ugent.be; daniel.dezutter@ugent.be). into series of orthogonal polynomials, suitably chosen depending on the probability distribution [4]. The unknown expansion coefficients are computed via the solution of an augmented system of equations, constructed using a stochastic Galerkin method (SGM). Pertinent statistical information is then readily obtained from these coefficients.

On the one hand, the technique has been used in [5] and [6] to analyze linear RLC networks. A more systematic and circuit-oriented approach was proposed in [7], where equivalent circuit models were proposed to model uncertainties in lumped linear electrical devices. The approach was later implemented in a customized circuit analysis tool having a (limited) capability of handling also nonlinear components [8]. However, an important limitation is that device nonlinearities are handled using either small-signal, linearized equivalent models, or approximate Taylor expansions. Furthermore, an ad-hoc software, with customized library models, is required, rather than relying on available and standard circuit solvers. Compatibility with commercial tools is a very desirable requirement for circuit designers, so that well-consolidated algorithms and device models can be exploited.

On the other hand, the authors of this paper proposed a PC-based modeling strategy for the statistical assessment of stochastic distributed networks in standard SPICE-type design environments [9]. Nonetheless, the methodology presented in [9] focuses exclusively on the variability provided by transmission-line elements with random properties, while lacking the inclusion of random lumped elements as well as of nonlinear components. The present paper covers this gap by outlining a very general framework for stochastic circuit simulation, consisting of random distributed and nonlinear lumped components. The latter include elements with userdefined I-V-characteristic or built-in device models, like those for diodes and MOSFETs available in many SPICE-type simulators. It is important to stress that the proposed modeling technique is fully compatible with standard and commerciallyavailable circuit solvers.

This paper is organized as follows: Section II outlines the basic principles of PC; Section III introduces the modeling of random linear circuit elements; Section IV describes the modeling of nonlinear devices; Section V outlines the general procedure for the PC-based simulation of stochastic networks; in Section VI, the proposed approach is illustrated and validated by means of application examples; finally, conclusions are drawn in Section VII. 


\section{Polynomial Chaos Overview}

In an electrical network where some elements are stochastic, voltages and currents also become stochastic. The underlying idea for the simulation of such a stochastic network is to expand voltages and currents into the spectral PC series [9], i.e.

$$
v(t, \boldsymbol{\xi}) \approx \sum_{k=0}^{P} v_{k}(t) \varphi_{k}(\boldsymbol{\xi}), \quad i(t, \boldsymbol{\xi}) \approx \sum_{k=0}^{P} i_{k}(t) \varphi_{k}(\boldsymbol{\xi}),
$$

where $\boldsymbol{\xi}=\left[\xi_{1}, \ldots, \xi_{d}\right]$ is a vector collecting the independent and normalized random variables (RVs) parameterizing all the variations (e.g., manufacturing tolerances and/or temperature fluctuations). Furthermore, $v_{k}(t)$ and $i_{k}(t)$ are deterministic voltage and current coefficients to be determined. Finally, $\left\{\varphi_{k}\right\}_{k=0}^{P}$ forms a basis of multivariate polynomial functions that are orthonormal with respect to the following inner product:

$$
\langle f, g\rangle=\int_{\mathbb{R}^{d}} f(\boldsymbol{\xi}) g(\boldsymbol{\xi}) w(\boldsymbol{\xi}) d \boldsymbol{\xi},
$$

$w(\boldsymbol{\xi})$ being the joint probability distribution of $\boldsymbol{\xi}$.

According to the Wiener-Askey scheme, for standard distributions, the orthogonal polynomials for $d=1$ are well-known and correspond to Hermite polynomials for Gaussian RVs, Legendre polynomials for uniform RVs, etc. [4]. It is important to point out that, in order to preserve symmetry and reciprocity of the models, we adopt here a normalized (i.e., rescaled) version of such polynomials [10], rendering $\left\langle\varphi_{k}, \varphi_{j}\right\rangle=\delta_{k j}$ (Kronecker's delta). When $d>1$, the multivariate polynomials are built as product combinations of univariate ones, in order to preserve their orthonormality [3].

The expansions (1) are truncated to a maximum total degree $p$ of the multivariate polynomials $\varphi_{k}$, so that the total number of terms is

$$
P+1=\frac{(p+d) !}{p ! d !} .
$$

As shown e.g. in [9], for a broad class of problems, choosing $p=2$ generally provides satisfactory modeling accuracy.

The main advantage of the PC representation (1) is that the spectral voltages and currents are deterministic, the randomness being confined into the polynomials. Thanks to the orthogonality properties, the first two statistical moments of the circuit variables are readily obtained as [3]

$$
\mathrm{E}\{v(t, \boldsymbol{\xi})\}=v_{0}(t), \quad \mathrm{E}\{i(t, \boldsymbol{\xi})\}=i_{0}(t)
$$

and

$$
\operatorname{Var}\{v(t, \boldsymbol{\xi})\}=\sum_{k=1}^{P} v_{k}^{2}(t), \quad \operatorname{Var}\{i(t, \boldsymbol{\xi})\}=\sum_{k=1}^{P} i_{k}^{2}(t) .
$$

Higher order moments as well as probability density functions (PDFs) are obtained by randomly sampling (1) in accordance with the distribution of $\boldsymbol{\xi}$. This post-processing procedure is very fast because (1) is merely a polynomial function.

\section{MODELING OF LiNEAR ELEMENTS}

As discussed in the previous section, the statistical information on the circuit response is readily obtained, provided that the spectral coefficients $v_{k}(t)$ and $i_{k}(t)$ of the node voltages and branch currents are known. In order to avoid repeated and time-consuming simulations of the stochastic network, in [9] pertinent equivalent circuit models are created, connected and simulated in a SPICE-type tool to retrieve $v_{k}(t)$ and $i_{k}(t)$.

Unfortunately, in [9], only (multiconductor) transmission lines with random cross-sectional properties in combination with deterministic linear lumped elements are discussed. In this paper, a far more general framework is outlined, in which both linear and nonlinear elements, possibly subject to variability themselves, can be included. The discussion starts from the basic linear elements, i.e. resistors, capacitors and inductors. The modeling of nonlinear devices is discussed in the next section.

\section{A. Resistors}

The current-voltage relationship of a resistor, shown in Fig. 1a, is governed by Ohm's law:

$$
i(t)=G\left(v^{+}(t)-v^{-}(t)\right)=G v(t),
$$

with $G=1 / R$. If the resistance $R$ is random, e.g. due to manufacturing tolerances or temperature variations, its conductance can be expressed with a PC expansion analogous to (1), i.e.

$$
G(\boldsymbol{\xi})=\frac{1}{R(\boldsymbol{\xi})} \approx \sum_{k=0}^{P} G_{k} \varphi_{k}(\boldsymbol{\xi}),
$$

and hence, the voltage and current in (6) become random themselves. Given the orthonormality of the polynomials, the coefficients $G_{k}$ are given by

$$
G_{k}=\left\langle G, \varphi_{k}\right\rangle=\int_{\mathbb{R}^{d}} \frac{1}{R(\boldsymbol{\xi})} \varphi_{k}(\boldsymbol{\xi}) w(\boldsymbol{\xi}) d \boldsymbol{\xi} .
$$

Introducing (7) and (1) into (6) yields

$$
\sum_{k=0}^{P} i_{k}(t) \varphi_{k}(\boldsymbol{\xi}) \approx \sum_{k=0}^{P} \sum_{j=0}^{P} G_{k} v_{j}(t) \varphi_{k}(\boldsymbol{\xi}) \varphi_{j}(\boldsymbol{\xi}) .
$$

Application of a SGM, i.e. multiplying the left- and righthand sides of (9) by $\varphi_{m}(\boldsymbol{\xi})$ and integrating them using the inner product (2), yields

$$
i_{m}(t)=\sum_{j=0}^{P} \tilde{G}_{m j} v_{j}(t)=\sum_{j=0}^{P} \tilde{G}_{m j}\left(v_{j}^{+}(t)-v_{j}^{-}(t)\right),
$$

where we defined

$$
\tilde{G}_{m j}=\sum_{k=0}^{P} G_{k} \alpha_{k j m}
$$

with $\alpha_{k j m}=\left\langle\varphi_{k} \varphi_{j}, \varphi_{m}\right\rangle$.

It is worthwhile noting that (10) is now a deterministic equation relating the $m$ th spectral current coefficient to all the spectral voltage coefficients and can be implemented in any circuit solver using the equivalent circuit illustrated in Fig. 1b. As shown in the figure, the terms $\tilde{G}_{m m}$ are still implemented 


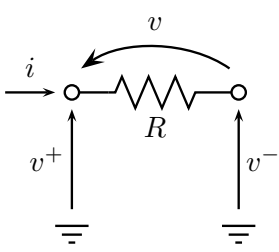

(a)

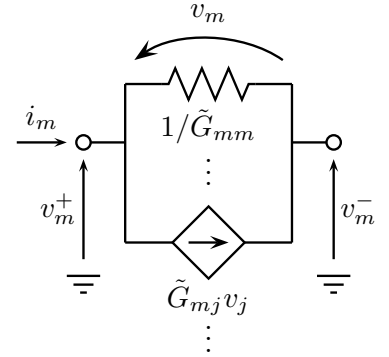

(b)
Fig. 1. Resistor element (a) and corresponding equivalent circuit for the $m$ th spectral current coefficient (b).

using a resistor, while the terms $\tilde{G}_{m j}$ (with $m \neq j$ ) correspond to voltage-dependent current sources, which provide the necessary coupling among the branches. By letting $m$ run from 0 to $P$, a set of $P+1$ deterministic equations is obtained, that correspond to the sought-for constitutive relationships linking all the spectral voltage and current coefficients.

\section{B. Capacitors}

The constitutive equation of a capacitor, shown in Fig. 2a, is

$$
i(t)=C \frac{d}{d t}\left(v^{+}(t)-v^{-}(t)\right)=C \frac{d}{d t} v(t) .
$$

Application of the SGM leads to

$$
i_{m}(t)=\sum_{j=0}^{P} \tilde{C}_{m j} \frac{d}{d t} v_{j}(t), \forall m=0, \ldots, P,
$$

with $\tilde{C}_{m j}=\sum_{k=0}^{P} C_{k} \alpha_{k j m}$. The equivalent circuit for the $m$ th spectral equation is shown in Fig. 2b. In this case, the coupling among the spectral coefficients is obtained via current-dependent current sources.

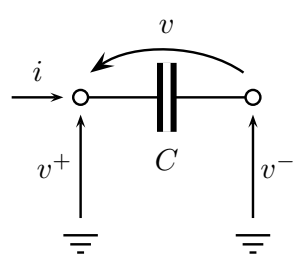

(a) (b)

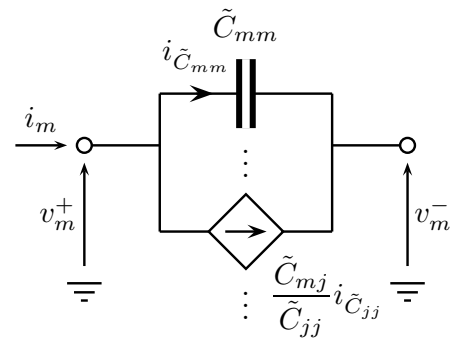

Fig. 2. Capacitor element (a) and corresponding equivalent circuit for the $m$ th spectral current coefficient (b)

\section{Inductors}

The behavior of an inductor, shown in Fig. 3a, is governed by the following equation

$$
v^{+}(t)-v^{-}(t)=v(t)=L \frac{d}{d t} i(t)
$$

The same SGM-based procedure used for resistors and capacitors leads to

$$
v_{m}(t)=v_{m}^{+}(t)-v_{m}^{-}(t)=\sum_{j=0}^{P} \tilde{L}_{m j} \frac{d}{d t} i_{j}(t),
$$

where once again $\tilde{L}_{m j}=\sum_{k=0}^{P} L_{k} \alpha_{k j m}$. The $m$ th spectral equation corresponds to the voltage across a coupled inductor and the pertinent equivalent circuit is illustrated in Fig. $3 \mathrm{~b}$.

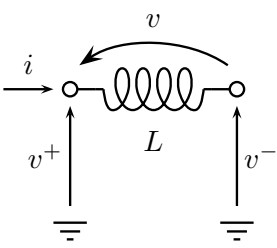

(a)

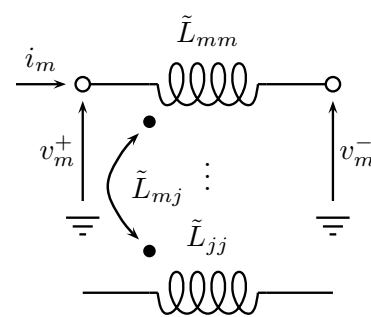

(b)
Fig. 3. Inductor element (a) and corresponding equivalent circuit for the $m$ th spectral voltage coefficient (b).

The equivalent circuits in Figs. 1-3 are readily implemented in any, e.g. SPICE-type, circuit simulator. However, it is important to remark that the augmented constitutive equations derived so far for resistors (10), capacitors (13) and inductors (15), were proven to describe passive models, independently of their specific circuit implementation [11]. So, adopting the new implementation as proposed in the present paper, guarantees stability during, e.g., transient simulations.

\section{Modeling of Nonlinear Elements}

When the governing equations are nonlinear, the derivation of the constitutive equations relating the voltage and current coefficients is hindered by the fact that, in general, no closedform expression exists for the integrals appearing in the SGM. A novel, efficient and SPICE-compatible modeling strategy to tackle this issue is outlined in this section, starting from the case of two-terminal nonlinear elements having a deterministic $\mathrm{I}-\mathrm{V}$-characteristic. The formulation is later extended to threeterminal elements (transistors) as well as to nonlinear elements with randomness in their characteristic.

\section{A. Deterministic Two-Terminal Devices}

Let us consider the case of a generic nonlinear conductance (Fig. 4a):

$$
i(t)=F\left(v^{+}(t)-v^{-}(t)\right)=F(v(t)) .
$$

Substitution of the expansions (1) into (16) yields

$$
\sum_{k=0}^{P} i_{k}(t) \varphi_{k}(\boldsymbol{\xi})=F\left(\sum_{k=0}^{P} v_{k}(t) \varphi_{k}(\boldsymbol{\xi})\right) .
$$

Application of the SGM produces, $\forall m=0, \ldots, P$,

$$
i_{m}(t)=\int_{\mathbb{R}^{d}} F\left(\sum_{k=0}^{P} v_{k}(t) \varphi_{k}(\boldsymbol{\xi})\right) \varphi_{m}(\boldsymbol{\xi}) w(\boldsymbol{\xi}) d \boldsymbol{\xi} .
$$


As anticipated, in general the integral in the right-hand side cannot be resolved in closed form, except when the nonlinear function $F(\cdot)$ is of the polynomial type [12].

When the function is not polynomial, but for example an exponential diode equation, a very general, approximate formulation to solve the issue has been presented in [13]. The integral in (18) is discretized by means of a quadrature rule $\forall m=0, \ldots, P$ :

$$
\begin{aligned}
i_{m}(t) & \approx \sum_{q=1}^{Q} F\left(\sum_{k=0}^{P} v_{k}(t) \varphi_{k}\left(\boldsymbol{\xi}_{q}\right)\right) \varphi_{m}\left(\boldsymbol{\xi}_{q}\right) w_{q} \\
& =\sum_{q=1}^{Q} j_{q}\left(t, v_{0}(t), \ldots, v_{P}(t)\right) a_{m q} w_{q},
\end{aligned}
$$

where we introduced the notation $a_{i j}=\varphi_{i}\left(\boldsymbol{\xi}_{j}\right)$ and

$$
j_{q}\left(t, v_{0}(t), \ldots, v_{P}(t)\right)=F\left(\sum_{k=0}^{P} v_{k}(t) a_{k q}\right) .
$$

In (19), $Q$ is the number of quadrature points, whereas $\boldsymbol{\xi}_{q}$ are predefined multivariate samples of the RVs $\boldsymbol{\xi}$ and $w_{q}$ are the corresponding weights, both depending on the quadrature rule. As such, $a_{i j}$ are merely (precomputable) coefficients, thus rendering (19) a deterministic equation. Such an equation involves linear combinations of all the voltage spectral coefficients in the argument of the nonlinear function, as well as linear combinations of the nonlinear currents. As shown in the application examples, by using proper Gaussian quadrature rules [14], a very good modeling accuracy can be achieved with a low number of quadrature points.

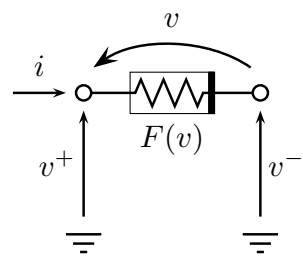

(a) (b)

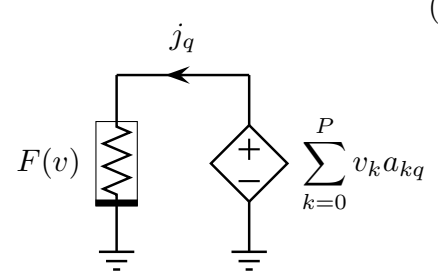

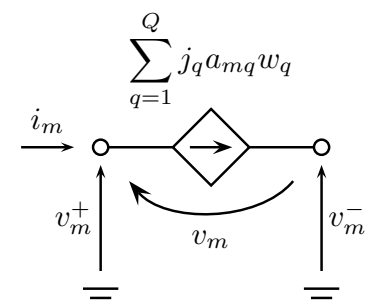

(c)
Fig. 4. Nonlinear conductance (a), illustration of the corresponding $q$ th companion cell (b) and equivalent circuit for the $m$ th spectral current coefficient (c).

When the nonlinear I-V-characteristic is known in analytical form, like for example the Schottky diode equation $i(t)=$ $F(v(t))=I_{s}\left(\exp \left(v(t) / V_{T}\right)-1\right)$, equations (19) could be implemented using behavioral dependent sources available in advanced circuit simulators. Nonetheless, we propose a more efficient and elegant circuit implementation of (19), which is also valid for user-defined and library elements with nonanalytical characteristics. This implementation is inspired by the observation that, for every $m$, the summation over $q$ always involves the same nonlinear terms $j_{q}(20)$. Therefore, the idea is to introduce an auxiliary, companion circuit cell responsible for the sampling of these nonlinear currents, as illustrated in Fig. 4b. A voltage-dependent voltage source is used to linearly combine the spectral voltage coefficients appearing in the argument of the nonlinear function. The nonlinear element with the pertinent I-V-characteristic $F(v(t))$ guarantees that the current $j_{q}$ flows in the companion circuit. In total, $Q$ such companion cells are defined and the $Q$ nonlinear currents $j_{q}$ are linearly combined in the main circuit by means of currentdependent current sources, as shown in Fig. 4c.

It is important to note that, according to this implementation, the overall circuit model involves the same type of nonlinearity $F(v)$ as the original circuit. This makes this implementation very efficient, despite the additional number of auxiliary nodes required by the companion cells. Moreover, as stated above, the nonlinear element can now be any user-defined device or library element (e.g., a diode) available in the solver, as there is no more need to have an explicit analytical I-V-characteristic.

\section{B. Three-Terminal Devices}

Reasoning along the same lines, three-terminal devices, like transistors, can also be dealt with. The structure of the companion cell and the spectral equivalent model are illustrated in Fig. 5 for a MOSFET. In this case, two voltage sources are required in the companion cell to linearly combine the input and output voltages, i.e. the gate-source and drain-source voltages, respectively. The resulting nonlinear gate and drain currents are then linearly combined in the spectral equivalent circuit by means of two dependent current sources. The current in the source terminal is determined by the Kirchhoff current law (KCL).

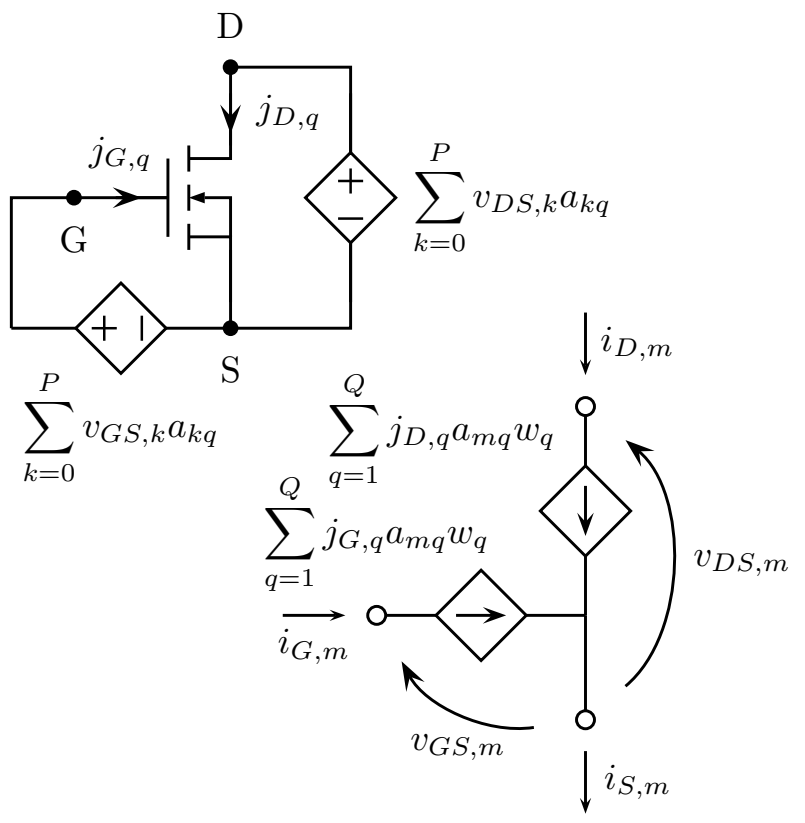

Fig. 5. Illustration of the $q$ th companion cell (top left) and the equivalent circuit for the $m$ th spectral current coefficients (bottom right) of a nonlinear three-terminal device. 


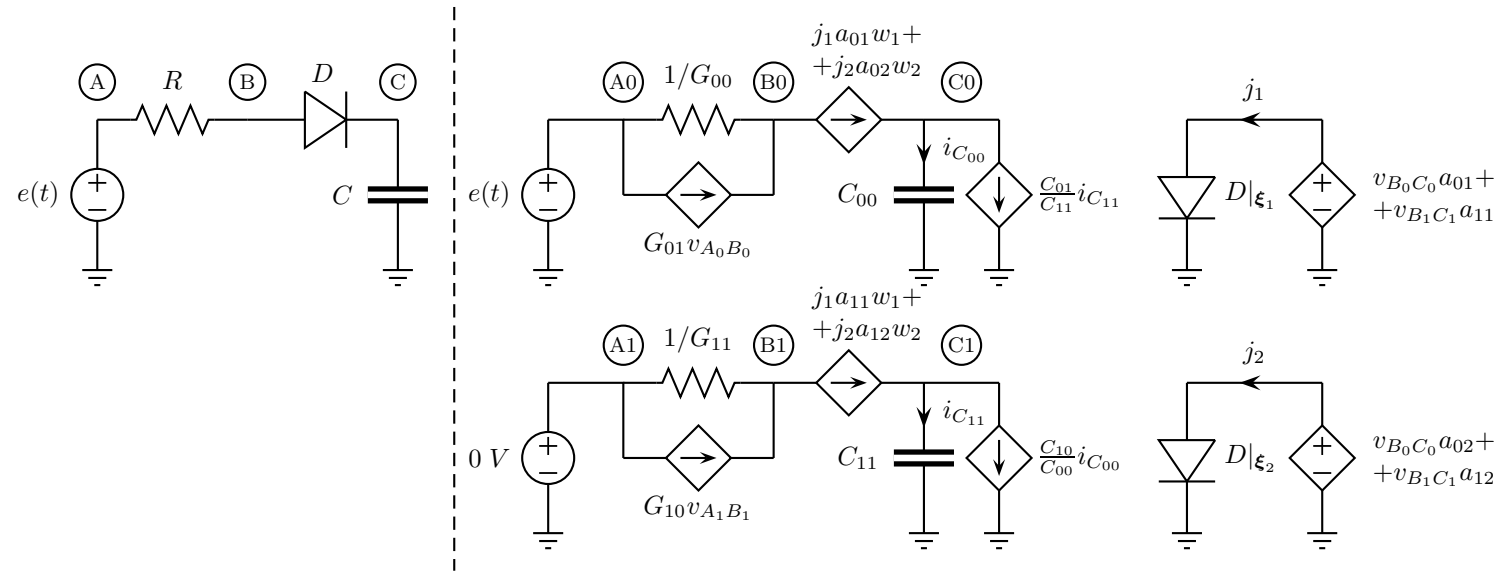

Fig. 6. Illustrative example: rectifier circuit. Left panel: original stochastic network; right panel: spectral circuits for $m=0$ and $m=1$ and pertinent companion cells for $q=1$ and $q=2$.

\section{Stochastic Nonlinear Devices}

If the nonlinear elements also exhibit variability, their characteristic inherently depends on the random vector $\boldsymbol{\xi}$, i.e. $F=F(v(t), \boldsymbol{\xi})$. As the integral in (18) is already sampled at the quadrature points $\boldsymbol{\xi}_{q}$, now the nonlinear currents (20) become

$$
j_{q}\left(t, v_{0}(t), \ldots, v_{P}(t)\right)=F \mid \boldsymbol{\xi}_{q}\left(\sum_{k=0}^{P} v_{k}(t) a_{k q}\right),
$$

where $\left.F\right|_{\boldsymbol{\xi}_{q}}=F\left(v(t), \boldsymbol{\xi}_{q}\right)$ denotes the sampled nonlinear characteristic. Again, the nonlinear circuit component may be simulated using the built-in device models. It suffices to define companion cells for each $\boldsymbol{\xi}_{q}$. This reasoning applies to both two- and three-terminal devices.

\section{Simulation Procedure}

Given the equivalent spectral circuit models introduced in the previous sections, the strategy for the PC-based simulation of a stochastic electrical network is as follows:

1) Assume node voltages and branch currents to be expanded according to (1).

2) For each component in the original network, build the $P+1$ spectral models as described in Sections III and IV.

3) Create a new augmented network, by associating a node to each voltage spectral coefficient, and properly connect these nodes in accordance with the original circuit topology.

4) Simulate the obtained network to calculate the soughtfor spectral voltage and current coefficients of the stochastic network, coinciding with the deterministic voltages and currents of the augmented network.

The above procedure can be easily automated and the inclusion of independent sources in the modeling is straightforward. For a deterministic voltage source $e(t)$, we get

$$
v(t)=v^{+}(t)-v^{-}(t)=e(t) .
$$

and application of the SGM produces

$$
v_{m}(t)=v_{m}^{+}(t)-v_{m}^{-}(t)=\left\{\begin{array}{ll}
e(t) & m=0 \\
0 & \forall m \neq 0
\end{array} .\right.
$$

A similar reasoning equivalently applies to independent current sources. Although not often done in practice, stochastic sources can be modeled as well.

Since now $P+1$ spectral coefficients are associated to each node voltage, for a stochastic circuit with $N$ nodes, the corresponding augmented network has an overall number of $(P+1) N$ nodes. Performing a single circuit simulation of this deterministic augmented network is, however, often much faster than running a large number of Monte Carlo simulations of the original stochastic network, as will be shown in the next section.

The outlined procedure is illustrated in Fig. 6, where a rectifier circuit with three nodes, namely $\mathrm{A}, \mathrm{B}$ and $\mathrm{C}$, is considered. For the sake of clarity, a PC expansion with only $P+1=2$ terms is assumed. Two spectral networks are therefore present. Moreover, a number $Q=2$ of companion cells are considered for the spectral model of the diode. The labels $\left.D\right|_{\xi_{1}}$ and $\left.D\right|_{\xi_{2}}$ attached to the diodes indicate that the random parameters of the diode model $D$ are sampled at the two quadrature points $\boldsymbol{\xi}_{1}$ and $\boldsymbol{\xi}_{2}$. Of course, the null excitation in Fig. 6, evolving from (23), could have been replaced by a short circuit.

\section{VALidation AND Numerical Results}

This section discusses three validation and application examples, illustrating the accuracy, efficiency and appositeness of the proposed simulation methodology. All the simulations are carried out with HSPICE on an ASUS U30S laptop with an Intel(R) Core(TM) i3-2330M, CPU running at $2.20 \mathrm{GHz}$ and 4 GB of RAM.

\section{A. Full-Bridge Diode Rectifier}

The first example considers the full-bridge diode rectifier shown in Fig. 7. The input signal $v_{\text {in }}$ is a sinusoidal voltage source with a peak amplitude of $5 \mathrm{~V}$ and a frequency of $60 \mathrm{~Hz}$. The load resistance and capacitance values are $R=1 \mathrm{k} \Omega$ and $C=1 \mathrm{mF}$, respectively. For the diodes, a standard SPICE model is used, having a series resistance of $1 \Omega$, a junction capacitance of $2 \mathrm{pF}$ and a saturation 


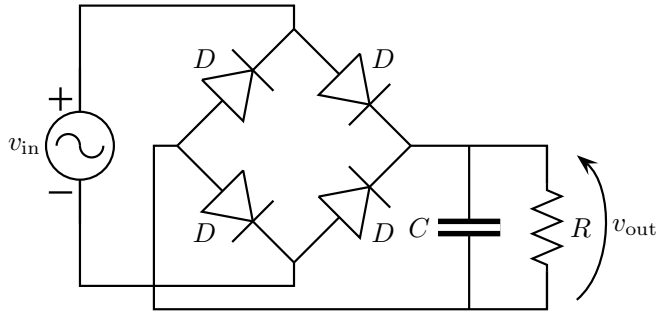

Fig. 7. Circuit schematic of the full-bridge diode rectifier.

current of $50 \mathrm{fA}$. The variability is provided by the operating temperature of the device, which uniformly varies in the range $[0,120]^{\circ} \mathrm{C}$. The temperature fluctuation affects both the diodes and the $R C$ load. For the latter, temperature coefficients of $\alpha_{R}=1500 \mathrm{ppm} /{ }^{\circ} \mathrm{C}$ and $\alpha_{C}=-750 \mathrm{ppm} /{ }^{\circ} \mathrm{C}$ are assumed for the resistor and the capacitor, respectively.

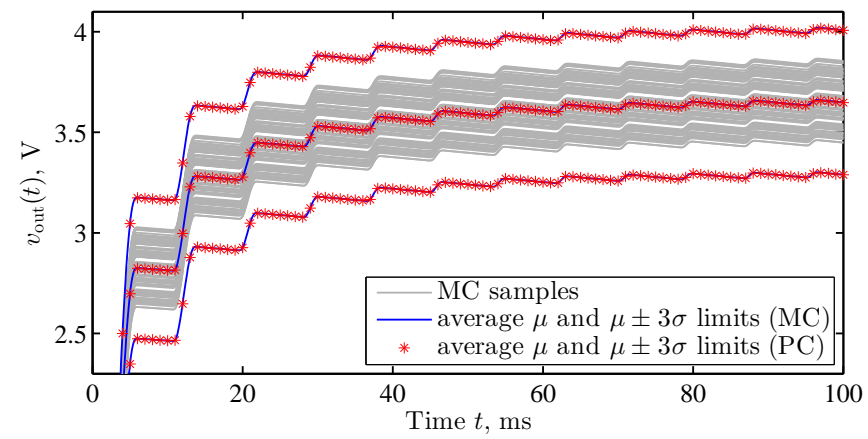

Fig. 8. Output voltage $v_{\text {out }}$ of the rectifier in Fig. 7. Gray lines: subset of 100 samples from Monte Carlo simulation; blue lines: average value $\mu$ and $\mu \pm 3 \sigma$ limits estimated with Monte Carlo analysis. The red asterisks indicate the same statistical information obtained from the PC expansion.

Fig. 8 shows the result of the stochastic simulation. The gray lines are a subset of 100 Monte Carlo samples providing a qualitative idea of the fluctuation of the rectifier output voltage $v_{\text {out }}$ due to temperature variations. The blue lines indicate the average response (hereafter denoted as $\mu$ ) as well as the average \pm three times the standard deviation (denoted as $\sigma$ ). These curves are estimated from 10000 Monte Carlo samples. Finally, the red markers denote the same statistical information obtained via the PC-based simulation of the deterministic spectral circuit, constructed considering $p=2$. For the modeling of the diodes, a Gauss-Legendre quadrature with $Q=3$ was considered. Excellent accuracy is established.

To assess the convergence of the Monte Carlo analysis, Fig. 9 shows the standard deviation of the rectifier output computed both from the PC coefficients and with an increasing number of Monte Carlo runs, i.e. 100, 1000 and 10000. The relative accuracy between the PC curve and the Monte Carlo estimation with 10000 samples is on the order of $0.1 \%$.

As far as the CPU time is concerned, the circuit simulation of the spectral network requires only $3 \mathrm{~s}$, whereas the Monte Carlo analysis takes about $42 \mathrm{~min}$ for 10000 runs. The speedup is thus $840 \times$ in this case.

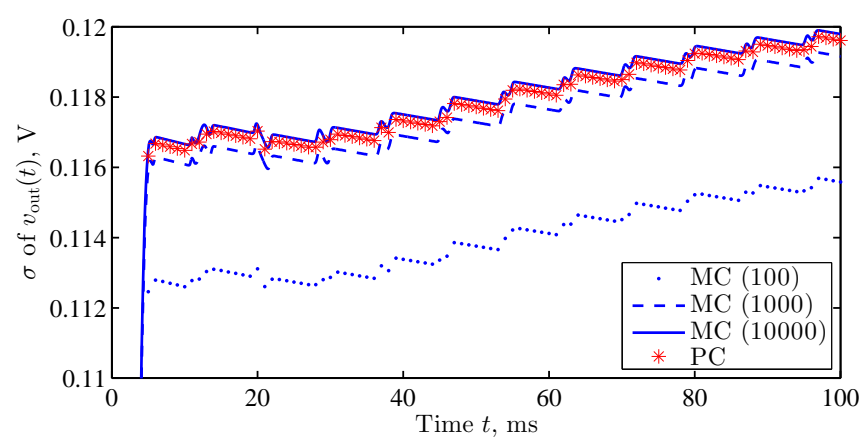

Fig. 9. Standard deviation of the rectifier output. Blue curves: results from Monte Carlo simulations with increasing number of samples; red asterisks: result from the PC-based approach.

\section{B. CMOS NAND Gate}

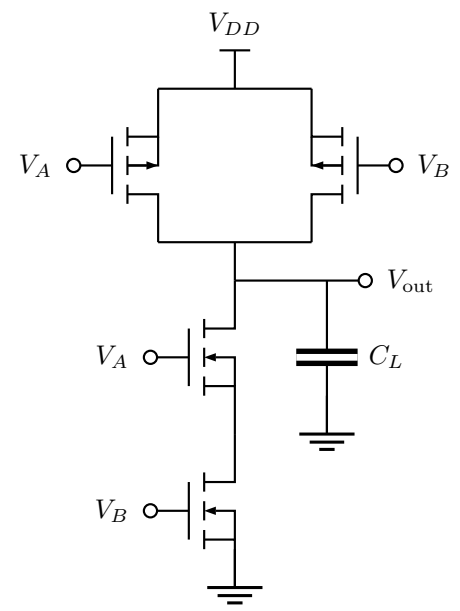

Fig. 10. Schematic of a two-input NAND gate in CMOS technology.

The second application example concerns a two-input NAND gate in CMOS technology, whose schematic is depicted in Fig. 10. The load capacitance $C_{L}$ models the input gate capacitance of the following stage and is considered as a Gaussian random variable with a mean value of $10 \mathrm{pF}$ and a relative standard deviation of $10 \%$. For the $n-$ and $p$ MOSFETs, a SPICE level-2 model is considered. Among the many parameters, a gate length of $1.2 \mu \mathrm{m}$, an oxide thickness of $20 \mathrm{~nm}$, and gate-drain and gate-source capacitances of $0.43 \mathrm{nF}$ are assumed. Finally, the power supply voltage is $V_{D D}=5 \mathrm{~V}$.

Fig. 11 shows the response of the logic gate for time varying inputs $V_{A}$ (solid black line) and $V_{B}$ (dashed gray line). The gray area shows the fluctuation of the response due to the load variability. The blue lines indicate the $\mu \pm 3 \sigma$ limits estimated after 10000 Monte Carlo runs, whilst the red markers show the same quantities obtained from the PC-based circuit simulation. For the latter, we used again $p=2$ and, for the transistors, a Gauss-Hermite quadrature with $Q=3$ was adopted. To assess the accuracy, a comparison of the standard deviation is provided in Fig. 12. Excellent agreement and a speed-up factor of about $1270 \times$ - arising from a PC-based analysis 


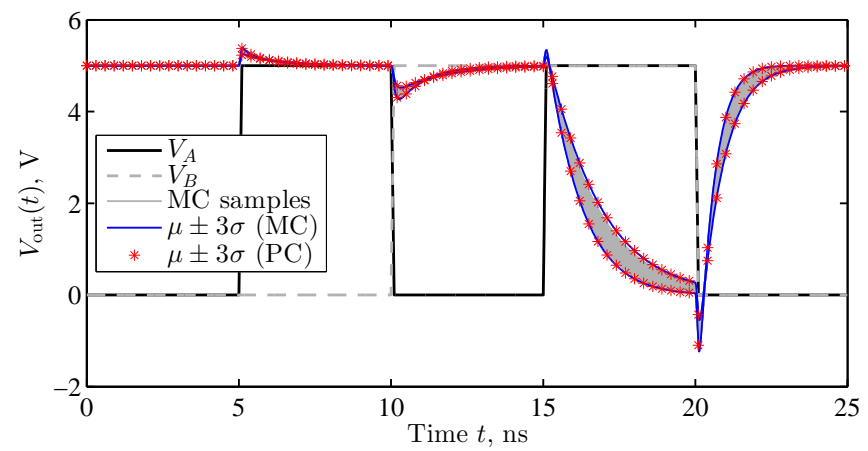

Fig. 11. Simulation result of the NAND gate in Fig. 10. Solid black line and dashed gray line: input gate voltages; gray area: subset of 100 samples of the output voltage from Monte Carlo simulation; blue lines and red asterisks: $\mu \pm 3 \sigma$ limits of the output estimated with Monte Carlo and PC, respectively.

taking $8.2 \mathrm{~s}$ against the $2 \mathrm{~h}$ and $54 \mathrm{~min}$ required by the Monte Carlo simulation - are achieved.

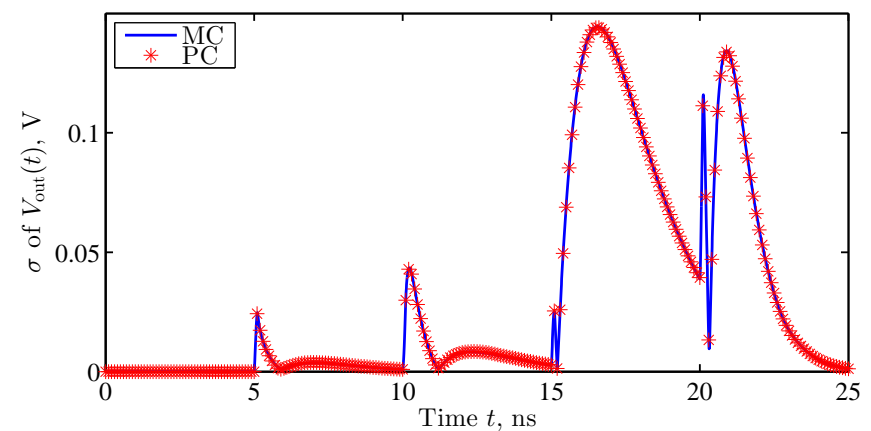

Fig. 12. Standard deviation of the NAND gate output voltage. Blue curve result from Monte Carlo simulation; red asterisks: result from the PC-based analysis

\section{Low-Noise Amplifier}

The third example deals with the low-noise amplifier depicted in Fig. 13, made up of three $0.25-\mu$ m-process $n$ MOSFETs. The RF input is a $1-\mathrm{GHz}$ sine wave with a peak amplitude of $1 \mathrm{~V}$ and a DC bias of $0.595 \mathrm{~V}$. The variability is induced by the process tolerance on the inductors, whose values are considered as two independent Gaussian RVs with means $L_{1}=13 \mathrm{nH}$ and $L_{2}=0.9 \mathrm{nH}$ and a relative standard deviation of $5 \%$. The values of the remaining circuit elements are $R_{S}=50 \Omega, R_{1}=400 \Omega, R_{2}=255 \Omega, R_{3}=120 \Omega$, and $V_{\text {bias }}=1.19 \mathrm{~V}$. The power supply voltage is $V_{D D}=2.3 \mathrm{~V}$ and the three MOSFET devices are simulated using accurate level-49 SPICE models. For the PC-based modeling of the MOSFETs, a bivariate Gauss-Hermite quadrature with $Q=9$ is adopted.

Fig. 14 provides the result of a stochastic simulation of the current $i_{L_{2}}$ flowing through inductor $L_{2}$. Again, the fluctuation of the current due to variability of the inductors is illustrated by means of Monte Carlo samples indicated in gray. The $\mu \pm 3 \sigma$ bounds obtained with both Monte Carlo and PC are also shown. A comparison of the standard deviation (see

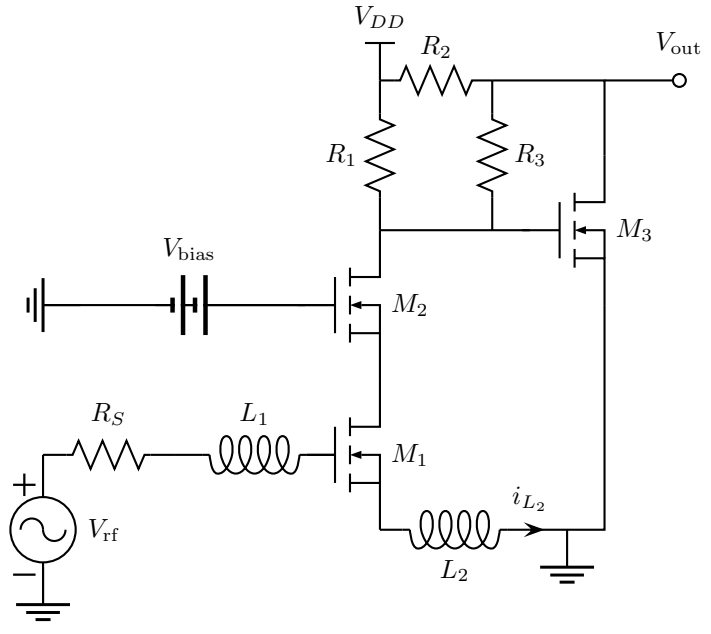

Fig. 13. Circuit schematic of the low-noise amplifier.

Fig. 15 and, in particular, the two close-ups) shows that, for a comparable accuracy between Monte Carlo and PC, up to 10000 runs are necessary, leading to a simulation time of about $40 \mathrm{~min}$. The simulation of the PC-based spectral circuit requires $4.6 \mathrm{~s}$, thus yielding a speed-up of $520 \times$.

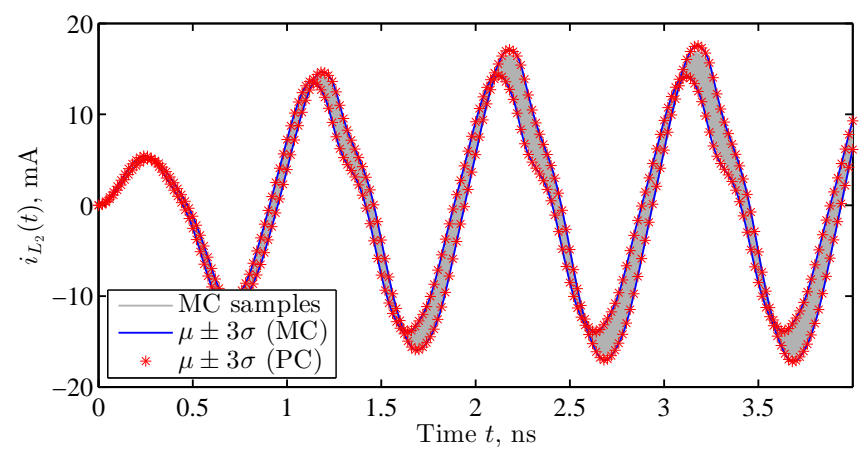

Fig. 14. Current $i_{L_{2}}$. Gray area: subset of 100 samples from Monte Carlo simulation; blue lines and red asterisks: $\mu \pm 3 \sigma$ limits of the output estimated with Monte Carlo and PC, respectively.

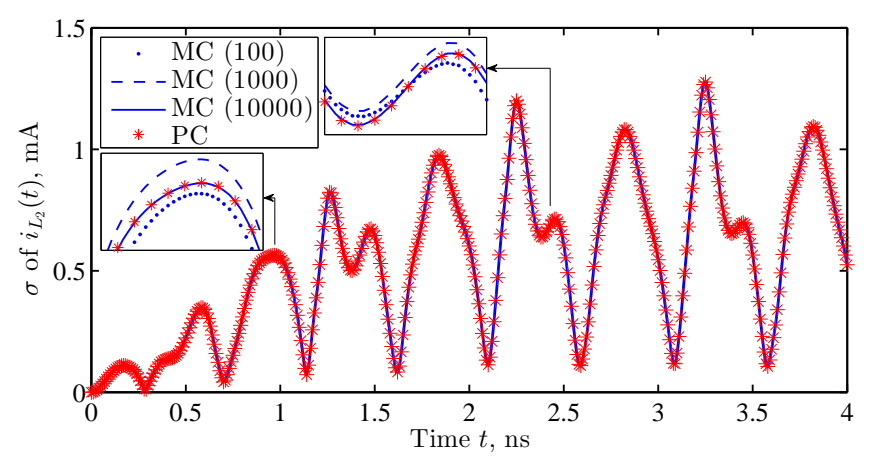

Fig. 15. Standard deviation of $i_{L_{2}}$. Blue curves: results from Monte Carlo analyses with increasing number of samples; red asterisks: result from the PC-based simulation.

From the PC expansion it is also possible to extract probability distributions. For instance, the PDF of $i_{L_{2}}$ at $t=3.12 \mathrm{~ns}$ 
is given in Fig. 16. The gray bars are the histogram obtained from the Monte Carlo samples, whilst the red line is the PDF evaluated from the PC expansion. Very good accuracy is established. The shape of this PDF differs substantially from a Gaussian distribution, therefore average and standard deviation are not sufficient to fully characterize the statistics of the circuit waveforms.

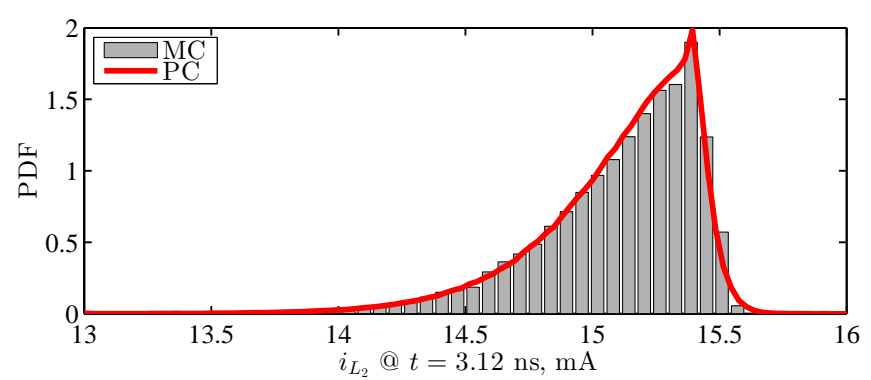

Fig. 16. Probability density function of $i_{L_{2}}(t)$ at $t=3.12 \mathrm{~ns}$. Gray bars estimation of Monte Carlo analysis. Red line: result from the PC expansion.

\section{CONCLUSIONS}

This paper outlines a systematic procedure for the efficient statistical simulation of general nonlinear circuits in standard SPICE-type design environments. More specifically, it shows that a stochastic circuit containing linear and nonlinear components, including nondeterministic two- and three-terminal devices, is converted into a single deterministic augmented circuit that is readily implemented in SPICE. The proposed procedure is based on the expansion of circuit voltages and currents in terms of PC series, from which the desired statistical information is quickly derived. The PC-based technique provides excellent accuracy and superior efficiency compared to standard sampling-based methods like Monte Carlo.

\section{REFERENCES}

[1] G. S. Fishman, Monte Carlo: Concepts, Algorithms, and Applications. New York: Springer-Verlag, 1996.

[2] A. H. Zaabab, Qi-Jun Zhang, and M. Nakhla, "A neural network modeling approach to circuit optimization and statistical design," IEEE Trans. Microw. Theory. Tech., vol. 43, no. 6, pp. 1349-1358, Jun. 1995.

[3] D. Xiu, "Fast numerical methods for stochastic computations: a review," Communications in Computational Physics, vol. 5, no. 2-4, pp. 242-272, Feb. 2009.

[4] D. Xiu and G. E. Karniadakis, "The Wiener-Askey polynomial chaos for stochastic differential equations," SIAM, Journal of Sci. Computation, vol. 24, no. 2, pp. 619-622, 2002.

[5] S. Vrudhula, J. M. Wang, and P. Ghanta, "Hermite polynomial based interconnect analysis in the presence of process variations," IEEE Trans. Comput. Aided Design Int. Circ. Syst., vol. 25, no. 10, pp. 2001-2011, Oct. 2006.

[6] N. Mi, S. X.-D. Tan, Y. Cai, and X. Hong, "Fast variational analysis of onchip power grids by stochastic extended Krylov subspace method," IEEE Trans. Comput. Aided Design Int. Circ. Syst., vol. 27, no. 11, pp. 19962006, Nov. 2008.

[7] Q. Su and K. Strunz, "Stochastic circuit modelling with Hermite polynomial chaos," IET Electronics Letters, vol. 41, no. 21, pp. 1163-1165, Oct. 2005.

[8] K. Strunz and Q. Su, "Stochastic formulation of SPICE-type electronic circuit simulation using polynomial chaos," ACM Trans. Model. Comput. Simul., vol. 18, no. 4, pp. 15:1-15:23, Sep. 2008.
[9] P. Manfredi, D. Vande Ginste, D. De Zutter, and F. G. Canavero, "Uncertainty assessment of lossy and dispersive lines in SPICE-type environments," IEEE Trans. Compon. Packag. Manuf. Techol., vol. 3, no. 7, pp. 1252-1258, Jul. 2013.

[10] P. Manfredi, D. Vande Ginste, D. De Zutter, and F. G. Canavero, "Improved polynomial chaos discretization schemes to integrate interconnects into design environments," IEEE Microw. and Wireless Compon. Lett., vol. 23, no. 3, pp. 116-118, Mar. 2013.

[11] P. Manfredi, D. Vande Ginste, D. De Zutter, and F. G. Canavero, "On the passivity of polynomial chaos-based augmented models for stochastic circuits," IEEE Trans. Circuits Syst. I, Reg. Papers, vol. PP, no. 99, pp. 110, 2013 (early access article).

[12] P. Manfredi, A. Biondi, D. Vande Ginste, D. De Zutter, and F. G. Canavero, "SPICE-based statistical assessment of interconnects terminated by nonlinear loads with polynomial characteristics," to appear in Proc. IEEE 22th Conf. Elect. Perform. Electron. Packag. Syst., San Jose, CA, Oct. 2013.

[13] A. Biondi, D. Vande Ginste, D. De Zutter, P. Manfredi, and F. G. Canavero, "Variability analysis of interconnects terminated by general nonlinear loads," IEEE Trans. Compon. Packag. Manuf. Techol., vol. 3, no. 7, pp. 1244-1251, Jul. 2013.

[14] G. H. Golub and J. H. Welsch, "Calculation of Gauss quadrature rules,' Mathematics of Computation, vol. 23, pp. 221-230, 1969. 